\title{
Schrittmacherimplantation oder Optimierung der Medikation?
}

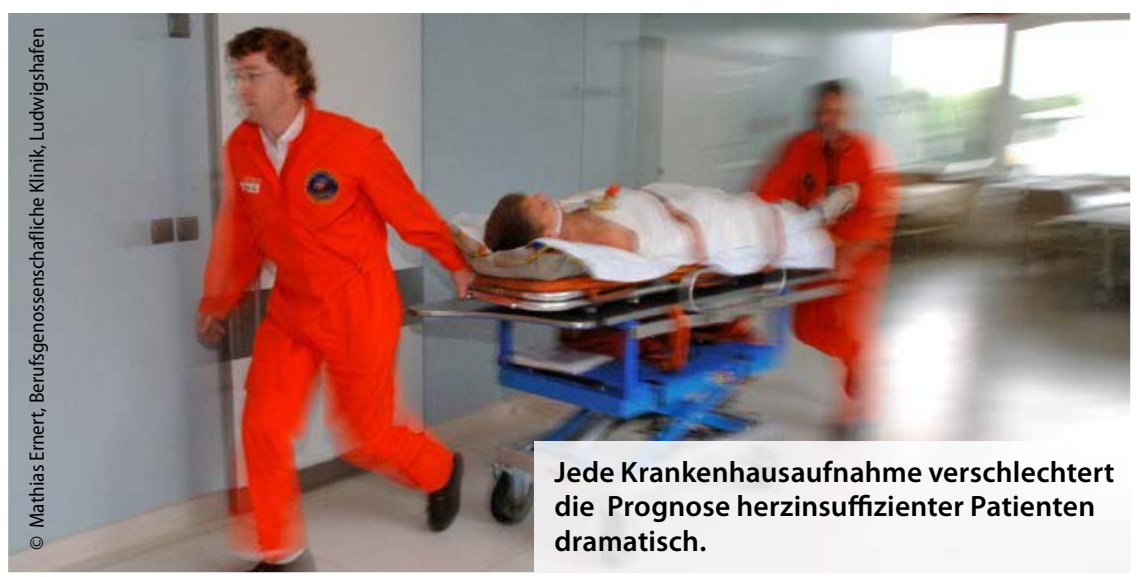

_ Die Prognose der chronischen Herzinsuffizienz ist schlecht. Nach einem ersten Krankenhausaufenthalt lebt ein herzinsuffizienter Patient im Durchschnitt nur noch 1,5 Jahre, berichtete Prof. Stephan Felix, Direktor der kardiologischen Universitätsklinik in Greifswald.

\section{Medikament oder Gerät?}

Die moderne Therapie bei diesem Krankheitsbild besteht heute aus Medikamenten und unterstützenden Geräten, z.B. biventrikulären Schrittmachern für die kardiale Resynchronisation (CRT). Durch eine optimale medikamentöse Therapie werde die Mortalität um fast $80 \%$ reduziert, mit einer additiven CRT sogar um 90\%. „Doch ein CRT-System sollte erst dann implantiert werden, wenn die medikamentöse Therapie voll ausgereizt ist und der Patient weiter symptomatisch ist", riet Felix.

Bei herzinsuffizienten Patienten mit einer funktionellen Mitralinsuffizienz steht heute mit dem MitraClip ein interventionelles Verfahren zur Verfügung. Symptome, aber für eine Lebenszeitverlängerung gibt es nach Felix keine ausreichende Evidenz.

\section{ARNI statt ACE-Hemmer}

Die primäre medikamentöse Herzinsuffizienztherapie sieht auch nach der neuen ESC-Leitlinie ACE-Hemmer bzw. $\mathrm{AT}_{1}$-Blocker, Betablocker und Mineralokortikoidrezeptorenblocker vor. Doch wenn der Patient unter dieser Therapie weiterhin symptomatisch ist, sollte der ACE-Hemmer bzw. $\mathrm{AT}_{1}$-Blocker gegen den Angiotensin-Rezeptor-NeprilysinInhibitor (ARNI) Sacubitril/Valsartan (Entresto ${ }^{\circledast}$ ) ausgetauscht werden.

„In der kürzlich aktualisierten Leitlinie hat diese Substanz eine Klasse-I-Empfehlung“, so Prof. Tim Süselbeck von der kardiologischen Praxisklinik in Ludwigshafen. Erste Erfahrungen mit dem ARNI seien sehr überzeugend. Bei vielen $\mathrm{Pa}$ tienten komme es rasch zu einer deutlichen Verbesserung der Symptome. Nur selten müsse die Dosis wegen einer Hy-
Dieser Eingriff verbessert zwar die potonie reduziert werden. Auch in der Zulassungsstudie sei eine Hypokaliämie bzw. eine Niereninsuffizienz unter Entresto ${ }^{\circ}$ seltener aufgetreten als unter einem ACE-Hemmer.

\section{Dr. Peter Stiefelhagen}

- Kardiologentage 2016; Berlin, September 2016 (Veranstalter: Novartis)

\section{Kurz notiert}

medi verlängert Vertrag mit dem Deutschen Skiverband $\rightarrow$ Der Hersteller von medizinischen Hilfsmitteln medi verlängert sein Engagement um weitere zwei Jahre bis April 2018. Damit stattet das Bayreuther Unternehmen rund 550 Athleten, Trainer, Betreuer, Mannschaftsärzte und Physiotherapeuten der Deutschen Ski-Nationalmannschaft z. B. mit Bandagen, Sportkompressionsbekleidung, igli-Carbon-Einlagen und medi-travelReisestrümpfen aus. CEP, die Sportmarke von medi, bleibt offizieller Ausrüster der Nationalmannschaften Biathlon und Ski Nordisch. medi-Produkte kommen auch über den Wettkampf hinaus zum Einsatz. „Nach dem Rennen ist es wichtig, dass man etwas für die Regeneration tut. Deshalb trage ich immer die CEP Recovery Socks", berichtet Simon Schempp, der mit der Biathlon-Staffel 2014 bei den Olympischen Winterspielen in Sotschi, Russland, die Silber-Medaille gewann. „Auch bei langen Flugreisen ziehe ich mediKompressionstrümpfe an und habe damit gute Erfahrungen gemacht", fügt er hinzu.

Neben dem Engagement als offizieller Ausrüster des DSV ist medi Hauptsponsor und Namensgeber des Basketball-Bundesligisten medi bayreuth sowie Sponsor des Schweizer Extremalpinisten Stephan Siegrist. 